\title{
A Statistical Evaluation of The Depth of Inheritance Tree Metric for Open-Source Applications Developed in Java
}

\author{
Sergiy Prykhodko ${ }^{1}$, Natalia Prykhodko ${ }^{2}$, Tetyana Smykodub ${ }^{1}$
}

\begin{abstract}
The Depth of Inheritance Tree (DIT) metric, along with other ones, is used for estimating some quality indicators of software systems, including open-source applications (apps). In cases involving multiple inheritances, at a class level, the DIT metric is the maximum length from the node to the root of the tree. At an application (app) level, this metric defines the corresponding average length per class. It is known, at a class level, a DIT value between 2 and 5 is good. At an app level, similar recommended values for the DIT metric are not known. To find the recommended values for the DIT mean of an app we have proposed to use the confidence and prediction intervals. A DIT mean value of an app from the confidence interval is good since this interval indicates how reliable the estimate is for the DIT mean values of all apps used for estimating the interval. A DIT mean value higher than an upper bound of prediction interval may indicate that some classes have a large number of the inheritance levels from the object hierarchy top. What constitutes greater app design complexity as more classes are involved. We have estimated the confidence and prediction intervals of the DIT mean using normalizing transformations for the data sample from 101 open-source apps developed in Java hosted on GitHub for the 0.05 significance level.
\end{abstract}

Keywords: statistical evaluation, software metric, depth of inheritance tree, opensource application, Java

\section{Introduction}

The Depth of Inheritance Tree (DIT) metric was firstly defined by Chidamber and Kemerer in [5]. The DIT metric, along with others, is used for estimating the faults [7], [19], [21],

\footnotetext{
${ }^{1}$ Department of Software of Automated Systems, sergiy.prykhodko@nuos.edu.ua

${ }^{2}$ Finance Department, Admiral Makarov National University of Shipbuilding, Heroes of Ukraine Ave., 9, Mykolaiv, 54025, Ukraine
} 
reusability [13] and other quality indicators [15] in software systems, including open-source ones [2], [8], [15], [21]. Additionally, the DIT metric is considered as a factor influencing the cost of testing [3], [20]. This metric provides for each class a measure of the inheritance levels from the object hierarchy top. In cases involving multiple inheritances, at a class level, the DIT metric is "the maximum length from the node to the root of the tree" [5]. A DIT value of 0 indicates a root. If there is a majority of DIT values below 2, it may represent poor exploitation of the advantages of object-oriented design (OOD) and inheritance. It is recommended that a maximum DIT value of 5 since more deep-rooted trees constitute greater design complexity as more methods and classes are involved [6]. However, there are apps in Java, in which the DIT values for some classes are significantly greater than 5. For example, in the well-known jEdit app version 5.5.0, the software metrics of which are analyzed in [15], out of 1390 classes, 179 ones have DIT values greater than 5. In this app, there are 32 classes that DIT values are more than twice the maximum recommended DIT value of 5, and the DIT values for the two classes are more than 10 times 5: 52 and 53 for the org.gjt.sp.jedit.pluginmgr.InstallPanel and org.gjt.sp.jedit.pluginmgr.ManagePanel respectively. In this regard, the question arises of how to evaluate the OOD of the entire app, and not its individual classes, from the point of view of the class hierarchy layout, if the DIT values of some classes are significantly greater than the maximum recommended DIT value of 5. In this case, the average inheritance depth (AID) can be used to determine the complexity of a program as a whole [14]. Like the AID metric at an app level, the DIT mean defines the average length from the node to the root of the tree per class, and the recommended values for the DIT mean are not known. To find the recommended values for the DIT mean of an app we have proposed to use the confidence and prediction intervals of the DIT mean as the complexity indicators of OOD of software apps from the point of view of the class hierarchy layout. A DIT mean value of an app from the confidence interval is good since this interval indicates how reliable the estimate is for the DIT mean values of all apps used for estimating the interval. A DIT mean value between the lower bounds of the confidence and prediction intervals, or between the upper bounds of the confidence and prediction intervals is also acceptable. A DIT mean value below the lower bound of the prediction interval may indicate poor exploitation of the advantages of OOD and inheritance. A DIT mean value higher than an upper bound of prediction interval may indicate that some classes have a large number of the inheritance levels from the object hierarchy top. What constitutes greater app design complexity as more classes and methods are involved.

We apply normalizing transformations to estimate the confidence and prediction intervals of the DIT mean by appropriate techniques. The technique for evaluating the prediction intervals is based on normalizing transformations and the Grubbs test. The technique is as follows [16]. Firstly, we normalize non-Gaussian data by the bijective normalizer transformation. Then we transform the sample mean of the non-Gaussian data using the normalizing transformation and calculate the deviation from the sample mean for normalized data in the Grubbs test. After that, we define the boundaries of the interval for normalized data by respectively subtracting and adding the calculated deviation from the sample mean. Finally, we detect the bounds of the prediction interval for non-Gaussian data by transforming the boundaries of the prediction interval for normalized data by the transformation inversed to normalizing one. The technique for estimating the confidence intervals of the sample mean is similar to the previous one, with the only difference that we 
define the deviation from the sample mean for normalized data by the Student $t$ distribution.

\section{The techniques to estimate the confidence and prediction intervals based on normalizing transformations}

The technique to estimate the prediction intervals is based on normalizing transformations and the Grubbs test [16]. At first, we normalize non-Gaussian random variable $X$ to Gaussian random variable $Z$ by a bijective normalizing transformation

$$
Z=\psi(X)
$$

which has the corresponding inverse transformation

$$
X=\psi^{-1}(Z)
$$

Then we transform the sample mean $\bar{x}$ of the non-Gaussian data using the transformation (1) as

$$
\bar{z}_{\bar{x}}=\psi(\bar{x})
$$

and define the deviation from the sample mean for normalized data in the Grubbs test

$$
\Delta_{z}=S_{z} \frac{N-1}{\sqrt{N}} \sqrt{\frac{t_{\alpha /(2 N), N-2}^{2}}{N-2+t_{\alpha /(2 N), N-2}^{2}}},
$$

where $N$ is the data size, $S_{z}$ is the sample standard deviation of the normalized data, $t_{\alpha /(2 N), N-2}$ is a Student $t$-distribution quantile with $N-2$ degrees of freedom and $\alpha /(2 N)$ significance level.

After that, we define the prediction interval bounds for normalized data by respectively subtracting and adding the calculated deviation (4) from the sample mean

$$
\left[\bar{z}_{\bar{x}}-\Delta_{z}, \bar{z}_{\bar{x}}+\Delta_{z}\right]
$$

and define the deviation from the sample mean for normalized data in the Grubbs test.

Finally, we detect the boundaries of the prediction interval for non-Gaussian data by transforming the bounds of the prediction interval for normalized data (5) using the inverse transformation (2)

$$
\left[\psi^{-1}\left(\bar{z}_{\bar{x}}-\Delta_{z}\right), \psi^{-1}\left(\bar{z}_{\bar{x}}+\Delta_{z}\right)\right] \text {. }
$$

The technique to evaluate the confidence intervals of the sample mean is similar to the previous one with the only difference that we define the deviation from the sample mean for normalized data by the Student $t$-distribution

$$
\Delta_{z}=t_{\alpha / 2, N-1} S_{z} / \sqrt{N}
$$


where $t_{\alpha / 2, N-1}$ is a Student $t$-distribution quantile with $N-1$ degrees of freedom and $\alpha / 2$ significance level.

Other formulae for calculating the confidence intervals of the sample mean are the same as (1)-(3), (5), and (6).

\section{Estimating the confidence and prediction intervals of the DIT mean for open-source apps developed in Java}

We have evaluated the confidence and prediction intervals of the DIT mean using normalizing transformations for the data sample from 101 open-source apps developed in Java hosted on GitHub (https://github.com/). We selected most downloaded apps of various types including frameworks and libs. The data sample was obtained using the CK tool (https://github.com/mauricioaniche/ck). All classes have DIT at least 1 since everyone inherits java.lang.Object. To make it happen, classes must exist in the app. The minimum and maximum values of the DIT mean equal to 1.0 and 3.5, respectively. The average and sample standard deviation values of the DIT mean are 1.640 and 0.3970 , respectively. Table 1 contains the observed and expected frequencies of the DIT mean values for seven intervals from 101 open-source apps developed in Java. For selecting the number of intervals, we used Sturges' rule and Scott's rule, which gave approximately the same result. Table 1 also presents the lower (LB) and upper (UB) bounds of seven DIT mean intervals for which the frequencies are shown. We used the Gaussian and Johnson distributions for calculating the expected frequencies of the DIT mean. The Johnson distributions for $S_{\mathrm{L}}, S_{\mathrm{B}}$, and $S_{\mathrm{U}}$ families in the form of the probability density functions are considered below.

Table 1. The observed and expected frequencies of DIT mean values

\begin{tabular}{|c|c|c|c|c|c|c|c|}
\hline \multirow{3}{*}{$j$} & \multicolumn{2}{|c|}{ DIT mean intervals } & \multirow{3}{*}{$\begin{array}{l}\text { Observed } \\
\text { frequen- } \\
\text { cies, } n_{j}\end{array}$} & \multicolumn{4}{|c|}{ Expected frequencies, $N p_{j}$} \\
\hline & \multirow{2}{*}{ LB } & \multirow{2}{*}{ UB } & & \multirow{2}{*}{ Gaussian } & \multicolumn{3}{|c|}{ Johnson } \\
\hline & & & & & $S_{\mathrm{L}}$ & $S_{\mathrm{B}}$ & $S_{\mathrm{U}}$ \\
\hline 1 & 1.000 & 1.357 & 24 & 18.64 & 24.89 & 24.22 & 23.81 \\
\hline 2 & 1.357 & 1.714 & 41 & 33.95 & 40.44 & 40.44 & 41.23 \\
\hline 3 & 1.714 & 2.071 & 24 & 29.01 & 22.01 & 22.71 & 22.39 \\
\hline 4 & 2.071 & 2.429 & 9 & 11.63 & 8.55 & 8.67 & 8.41 \\
\hline 5 & 2.429 & 2.786 & 2 & 2.18 & 3.01 & 2.90 & 2.87 \\
\hline 6 & 2.786 & 3.143 & 0 & 0.19 & 1.04 & 0.93 & 0.97 \\
\hline 7 & 3.143 & 3.500 & 1 & 0.01 & 0.37 & 0.30 & 0.34 \\
\hline
\end{tabular}

The null hypothesis $H_{0}$ that the observed frequency distribution of the DIT mean values is the same as the normal distribution was checked by the Pearson chi-squared test, according to which, at first we determine the chi-squared test statistic $\chi^{2}$

$$
\chi^{2}=\sum_{j=1}^{m} \frac{\left(n_{j}-N p_{j}\right)^{2}}{N p_{j}},
$$


where $m$ is the number of cells or observation intervals, $n_{j}$ is the number of observations of type $j, N$ is a total number of observations, $N p_{j}$ is the expected count of type $j$, approved by the null hypothesis that the share of type $j$ in the population is the probability $p_{j}$. Then we define the degrees of freedom (df) of that statistic. When the model is fully specified, computing the $\mathrm{df}$ is easy: $\mathrm{df}$ is equal to $m$ minus one [9]. For testing goodness of fit, the $\mathrm{df}$ reduced by the number of fitted parameters in the distribution, which for the Gaussian distribution equals two.

Further, we select a significance level for the result of the test. Finally, we compare $\chi^{2}$ to the critical value from the chi-squared distribution with df degrees of freedom and the selected confidence level. We reject the null hypothesis $H_{0}$ (there is no difference between the distributions) if the $\chi^{2}$ test statistic surpasses the critical value from the chi-squared distribution, and the alternative hypothesis $H_{1}$ (there is a difference between the distributions) can be taken, both with the selected confidence level. If the $\chi^{2}$ test statistic falls below the critical value from the chi-squared distribution, then no clear conclusion can be obtained, and the null hypothesis is sustained, but not necessarily accepted.

We have rejected the null hypothesis that the distribution of the DIT mean values is the same as the normal distribution and accept the alternative hypothesis $H_{1}$ (there is a difference between the distributions) since the chi-squared test statistic $\chi^{2}$ value equals to 21.34 is higher than the critical value of the chi-square, which equals to 9.49 for 4 degrees of freedom and 0.05 significance level. For the distribution of the DIT mean values, estimators of skewness and kurtosis equal to 1.36 and 6.49 , respectively. These values also show to us that the data of DIT mean are not Gaussian, since the skewness and kurtosis the Gaussian distribution equal to 0 and 3, respectively.

Therefore, we use normalizing transformations to evaluate the confidence and prediction intervals of the DIT mean. As in [18], for normalizing the data sample, we use the Johnson translation system that set up a transformation of a continuous random variable $X$ to a standard Gaussian variable $Z$ and is determined by

$$
Z=\gamma+\eta h(X, \varphi, \lambda) \sim N(0,1),
$$

where the translation function $h$ takes four possible forms

$$
h=\left\{\begin{array}{cl}
\ln (y), & \text { for } S_{L} \text { (lognormal)family; } \\
\ln [y /(1-y)], & \text { for } S_{B} \text { (bounded)family; } \\
\operatorname{Arsh}(y), & \text { for } S_{U} \text { (unbounded)family; } \\
y & \text { for } S_{N} \text { (normal)family }
\end{array}\right.
$$

Here $y=(X-\varphi) / \lambda ; \operatorname{Arsh}(y)=\ln \left(y+\sqrt{y^{2}+1}\right)$.

We try to apply the translation function $h$ for $S_{\mathrm{L}}, S_{\mathrm{B}}$, and $S_{\mathrm{U}}$ families since the skewness and kurtosis estimators for the observed frequency distribution of the DIT mean values equal to 1.36 and 6.49 , respectively. The parameter vector $\boldsymbol{\theta}=\{\gamma, \eta, \varphi, \lambda\}$ of (8) for $S_{\mathrm{B}}$ and $S_{\mathrm{U}}$ families are evaluated by the maximum likelihood method 


$$
\hat{\boldsymbol{\theta}}=\arg \max _{\boldsymbol{\theta}} l(X, \boldsymbol{\theta})
$$

where the log-likelihood function is

$$
l(X, \boldsymbol{\theta})=-\frac{N \ln (2 \pi)}{2}+\sum_{i=1}^{N} \ln \left[h^{\prime}\left(y_{i}\right)\right]-\frac{1}{2} \sum_{i=1}^{N} z_{i}^{2} .
$$

Here $h^{\prime}(\cdot)$ is the derivative of the function (9) with respect to $X$ for $S_{B}$ or $S_{\mathrm{U}}$ families; $z_{i}$ is the $i$-value of $Z$ from (8).

The Johnson transformation (8) for $S_{\mathrm{B}}$ family is also defined by [12]

$$
Z=\gamma+\eta \ln \frac{X-\varphi}{\varphi+\lambda-X}
$$

where

$$
\varphi<X<\varphi+\lambda, \eta>0, \lambda>0
$$

Given the formula (12), we may write (11) in the form

$$
\begin{gathered}
l(X, \boldsymbol{\theta})=N \ln (\eta \lambda)-\frac{N \ln (2 \pi)}{2}-\sum_{i=1}^{N} \ln \left(x_{i}-\varphi\right)-\sum_{i=1}^{N} \ln \left(\varphi+\lambda-x_{i}\right)- \\
-\frac{1}{2} \sum_{i=1}^{N}\left[\gamma+\eta \ln \frac{x_{i}-\varphi}{\varphi+\lambda-x_{i}}\right]^{2},
\end{gathered}
$$

where $x_{i}$ is the $i$-value of $X$ from (12).

The log-likelihood function (14) is also obtained taking into account that the Johnson probability density function (pdf) for $S_{\mathrm{B}}$ family has the form [12]

$$
f_{B}(X)=\frac{\eta \lambda}{\sqrt{2 \pi}(X-\varphi)(\lambda+\varphi-X)} \exp \left\{-\frac{1}{2}\left[\gamma+\eta \ln \left(\frac{X-\varphi}{\lambda+\varphi-X}\right)\right]^{2}\right\}
$$

where $\varphi<X<\varphi+\lambda,-\infty<\gamma<\infty, \eta>0,-\infty<\varphi<\infty, \lambda>0$.

Parameters of the transformation (12) were estimated by the maximum likelihood method (10) for the function (14) with restrictions (13). Estimators for parameters of the transformation (12) and pdf (15) are: $\hat{\gamma}=15.24612, \hat{\eta}=2.64089, \hat{\varphi}=0.6343678$ and $\hat{\lambda}=302.0811$. The value of the log-likelihood function (14) equals to -38.25 .

The Johnson transformation (8) for $S_{\mathrm{U}}$ family is also defined by [12]

$$
Z=\gamma+\eta \operatorname{Arsh}\left(\frac{X-\varphi}{\lambda}\right)
$$

where 


$$
\eta>0, \lambda>0
$$

Given the formula (16), we may write (11) in the form

$$
\begin{aligned}
l(X, \boldsymbol{\theta})=N & \ln (\eta)-\frac{N \ln (2 \pi)}{2}-\frac{1}{2} \sum_{i=1}^{N} \ln \left[\left(x_{i}-\varphi\right)^{2}+\lambda^{2}\right]- \\
& -\frac{1}{2} \sum_{i=1}^{N}\left[\gamma+\eta \operatorname{Arsh}\left(\frac{x_{i}-\varphi}{\lambda}\right)\right]^{2},
\end{aligned}
$$

where $x_{i}$ is the $i$-value of $X$ from (16).

The log-likelihood function (18) is also obtained taking into account that the Johnson pdf for $S_{\mathrm{U}}$ family has the form [12]

$$
f_{U}(X)=\frac{\eta}{\sqrt{\left.2 \pi(X-\varphi)^{2}+\lambda^{2}\right\}}} \exp \left\{-\frac{1}{2}\left[\gamma+\eta \ln \left(\frac{X-\varphi}{\lambda}+\sqrt{\left(\frac{X-\varphi}{\lambda}\right)^{2}+1}\right)\right]^{2}\right\}
$$

where $-\infty<X<\infty,-\infty<\gamma<\infty, \eta>0,-\infty<\varphi<\infty, \lambda>0$.

Parameters of the transformation (16) were evaluated by the maximum likelihood method (10) for the function (18) with restrictions (17). Estimators for parameters of the transformation (16) and pdf (19) are: $\hat{\gamma}=-3.31347, \hat{\eta}=2.27082, \hat{\varphi}=0.86354$ and $\hat{\lambda}=0.346336$. The value of the log-likelihood function (18) equals to -38.21 .

The Johnson transformation (8) for $S_{\mathrm{L}}$ family is also defined by [12]

$$
Z=\gamma+\eta \ln \left(\frac{X-\varphi}{\lambda}\right)
$$

where $X>\varphi,-\infty<\gamma<\infty, \eta>0,-\infty<\varphi<\infty, \lambda>0$.

The Johnson pdf for $S_{\mathrm{L}}$ family has the form [12]

$$
f_{L}(X)=\frac{\eta}{\sqrt{2 \pi}(X-\varphi)} \exp \left\{-\frac{\eta^{2}}{2}\left[\frac{\gamma-\eta \ln \lambda}{\eta}+\ln (X-\varphi)\right]^{2}\right\},
$$

where $X>\varphi,-\infty<\gamma<\infty, \eta>0,-\infty<\varphi<\infty, \lambda>0$.

Parameters of the transformation (20) were estimated from the solution of a system of nonlinear algebraic equations [12]

$$
\begin{aligned}
\varphi+\lambda \omega \rho= & \hat{\alpha}_{1} ; \lambda^{2} \omega^{2} \rho^{2}\left(\omega^{2}-1\right)=\hat{\mu}_{2} ; \lambda^{3} \omega^{3} \rho^{3}\left(\omega^{6}-3 \omega^{2}+2\right)=\hat{\mu}_{3} ; \\
& \lambda^{4} \omega^{4} \rho^{4}\left(\omega^{2}-1\right)^{2}\left(\omega^{8}+2 \omega^{6}+3 \omega^{4}-3\right)=\hat{\mu}_{4},
\end{aligned}
$$


where $\omega=\exp \left(0,5 / \eta^{2}\right), \rho=\exp (-\gamma / \eta) \quad \hat{\alpha}_{1}$ is the sample mean of the random variable $X$; $\hat{\mu}_{2}, \hat{\mu}_{3}$ and $\hat{\mu}_{4}$ are the estimators of central statistical moments of the second, third, and fourth orders of the random variable $X$. In our case, the values of $\hat{\alpha}_{1}, \hat{\mu}_{2}, \hat{\mu}_{3}$ and $\hat{\mu}_{4}$ equal to $1.640,0.1576,0.16114$, and 0.08508 , respectively.

Estimators for parameters of the transformation (20) and pdf (21) are: $\hat{\gamma}=2.97489$, $\hat{\eta}=2.41164, \hat{\varphi}=0.711132$ and $\hat{\lambda}=2.92978$.

The null hypotheses $H_{0}$ that there is no difference between the observed frequency distribution of the DIT mean values and the Johnson distribution for $S_{\mathrm{L}}, S_{\mathrm{B}}$, and $S_{\mathrm{U}}$ families were checked by the Pearson chi-squared test. The null hypotheses $H_{0}$ are sustained since its $\chi^{2}$ test statistic values are $2.69,1.24$, and 1.07 , respectively for $S_{\mathrm{L}}, S_{\mathrm{B}}$, and $S_{\mathrm{U}}$ families, fall below the critical value from the chi-squared distribution, which equals to 5.99 for 0.05 significance level and 2 degrees of freedom. The expected frequencies of the Johnson distributions for $S_{\mathrm{L}}, S_{\mathrm{B}}$, and $S_{\mathrm{U}}$ families are shown in Table 1.

Also, we normalize the data sample by the Box-Cox transformation [4]

$$
Z=x(\lambda)=\left\{\begin{array}{lll}
\frac{X^{\lambda}-1}{\lambda}, & \text { if } \lambda \neq 0 ; \\
\ln (X), & \text { if } \lambda=0 .
\end{array}\right.
$$

Here $\lambda$ is a parameter of the transformation (22).

The parameter of the Box-Cox transformation (22) is estimated by the maximum likelihood method [11]

$$
\hat{\lambda}=\arg \max _{\lambda} l(X, \lambda)
$$

where the log-likelihood function is

$$
l(X, \lambda)=C-\frac{N}{2} \ln \sum_{i=1}^{N} \frac{\left[x_{i}(\lambda)-\bar{x}(\lambda)\right]^{2}}{N}+(\lambda-1) \sum_{i=1}^{N} \ln \left(x_{i}\right) .
$$

Here $C$ is a constant, which is determined from the normalization condition; $\bar{x}(\lambda)=\sum_{i=1}^{N} x_{i}(\lambda) / N ; x_{i}(\lambda)$ is the $i$-value of $x(\lambda)$ or $Z$ from (22).

The parameter $\lambda$ of the transformation (22) was estimated by the maximum likelihood method (23) for the function (24). The parameter estimator $\hat{\lambda}$ is $-0,696336$. The value of the log-likelihood function (24) equals to 105.08 .

We apply the Pearson chi-squared test to check the normality of normalized data based on the Box-Cox and Johnson transformations for $S_{\mathrm{L}}, S_{\mathrm{B}}$, and $S_{\mathrm{U}}$ families. The Chi-square test has allowed us to confirm the null hypotheses that are compatible with the assumption of normality in normalized data based on the transformations (12), (16), (20), and (22), since its $\chi^{2}$ values, which equal to $1.236,1.065,2.041$ and 0.999 , respectively, are less than the critical value of the chi-square for 0.05 significance level and 4 degrees of 
freedom. The skewness and kurtosis estimators for the distribution of normalized data based on the transformation (12), (16), (20), (22) equal to 0.017 and $3.144,0.003$, and $2.984,-0.109$ and $3.224,0.005$ and 2.910 , respectively. These values also indicate the normality of the normalized data.

Table 2 shows the observed and expected frequencies of the normalized DIT mean values, which are transformed by the Box-Cox and Johnson transformations. Table 2 also contains the lower (LB) and upper (UB) bounds of seven actual and normalized DIT mean intervals for which the frequencies are calculated.

Table 2. The observed and expected frequencies of normalized DIT mean values

\begin{tabular}{|c|c|c|c|c|c|c|c|c|c|c|c|c|}
\hline \multirow[b]{3}{*}{$j$} & \multicolumn{8}{|c|}{ The Johnson transformation } & \multirow{2}{*}{\multicolumn{4}{|c|}{$\begin{array}{l}\text { The Box-Cox } \\
\text { transformation }\end{array}$}} \\
\hline & \multicolumn{4}{|c|}{$S_{\mathrm{B}}$} & \multicolumn{4}{|c|}{$S_{\mathrm{U}}$} & & & & \\
\hline & LB & UB & $n_{j}$ & $N p_{j}$ & LB & UB & $n_{j}$ & $N p_{j}$ & LB & UB & $n_{j}$ & $N p_{j}$ \\
\hline 1 & -2.489 & -1.709 & 4 & 3.77 & -2.440 & -1.680 & 4 & 3.95 & 0.000 & 0.119 & 4 & 3.95 \\
\hline 2 & -1.709 & -0.929 & 13 & 13.40 & -1.680 & -0.920 & 13 & 13.36 & 0.119 & 0.239 & 13 & 13.09 \\
\hline 3 & -0.929 & -0.149 & 28 & 26.69 & -0.920 & -0.160 & 28 & 26.02 & 0.239 & 0.358 & 27 & 25.42 \\
\hline 4 & -0.149 & 0.631 & 29 & 29.81 & -0.160 & 0.600 & 29 & 29.22 & 0.358 & 0.478 & 30 & 28.94 \\
\hline 5 & 0.631 & 1.410 & 17 & 18.68 & 0.600 & 1.360 & 17 & 18.93 & 0.478 & 0.597 & 17 & 19.31 \\
\hline 6 & 1.410 & 2.190 & 9 & 6.56 & 1.360 & 2.120 & 9 & 7.07 & 0.597 & 0.716 & 9 & 7.55 \\
\hline 7 & 2.190 & 2.970 & 1 & 1.29 & 2.120 & 2.880 & 1 & 1.52 & 0.716 & 0.836 & 1 & 1.73 \\
\hline
\end{tabular}

We have estimated the confidence, and prediction intervals of the DIT mean for a significance level of 0.05 by (6) using the transformations (12), (16), (20), and (22). Table 3 contains the width, LB and UB of the confidence and prediction intervals of the DIT mean for a significance level of 0.05 , which evaluated by (6) using the transformations (12), (16), (20), and (22). Also, we have estimated the confidence and prediction intervals of the DIT mean for a significance level of 0.05 by (5) without the normalization. The only difference being that we substitute the sample mean $\bar{x}$ instead of $\bar{z}_{\bar{x}}$ in (5), and for calculating $\Delta_{z}$ by (4) and (7), we use the sample standard deviation value of the DIT mean instead of $S_{z}$. Also, Table 3 contains the width, LB and UB bounds of the confidence and prediction intervals of the DIT mean for a significance level of 0.05 in this case, when we consider our data to be Gaussian. In this case, borders are symmetrical around the average. The bounds of the confidence and prediction intervals that are defined using the transformations (12), (16), (20), and (22) are not symmetrical around the average.

Also, according to the Grubbs test [10], the upper bound of the prediction interval equaled to 2.985 indicates there is one outlier in the data sample, for which the DIT mean value is 3.500. In the case of applying the normalizing transformations [16], [17], there are no outliers in the data sample for all considered transformations (12), (16), (20), and (22).

Easy to notice, the values of confidence interval bounds are similar. However, the prediction interval bounds based on the normalizing transformations and without one differ significantly. This result may be explained best fitting of the non-Gaussian data distribution by the pdfs (15), (19), and (21) than in the case of application of the normal distribution. 
Table 3. The confidence and prediction intervals of the DIT mean

\begin{tabular}{|l|c|c|c|c|c|c|}
\hline \multirow{2}{*}{ Transformations } & \multicolumn{3}{|c|}{ Confidence intervals } & \multicolumn{3}{c|}{ Prediction intervals } \\
\cline { 2 - 7 } & LB & UB & Width & LB & UB & Width \\
\hline The Johnson $S_{\mathrm{B}}$ & 1.568 & 1.718 & 0.150 & 0.914 & 4.230 & 3.316 \\
\hline The Johnson $S_{\mathrm{U}}$ & 1.569 & 1.717 & 0.148 & 0.883 & 4.471 & 3.558 \\
\hline The Johnson $S_{\mathrm{L}}$ & 1.567 & 1.719 & 0.152 & 0.939 & 4.496 & 3.557 \\
\hline The Box-Cox & 1.570 & 1.716 & 0.146 & 0.889 & 4.870 & 3.981 \\
\hline The normal & 1.562 & 1.719 & 0.157 & 0.295 & 2.985 & 2.690 \\
\hline
\end{tabular}

We have selected the confidence, and prediction intervals of the DIT mean for a significance level of 0.05 based on the Johnson transformation for $S_{\mathrm{U}}$ family (16) since only this one is the bijective normalizing transformation. In this case, a DIT mean value from 1.57 to 1.72 is good since specified values are the lower and upper bounds of the confidence interval. A DIT mean value from 0.88 to 1.57 or from 1.72 to 4.47 is also acceptable since specified values are the lower bounds of the confidence and prediction intervals, and the upper bounds of the confidence and prediction intervals respectively. A DIT mean value below 0.88 may indicate poor exploitation of the advantages of OOD and inheritance. A DIT mean value higher than 4.47 may indicate that some classes have a large number of the inheritance levels from the object hierarchy top.

We have compared these results with the DIT mean values of three popular open-source apps developed in Java: FreeMind, TuxGuitar, and jEdit having over 465k, 131k, and 56k downloads in 2019, respectively [15]. The DIT mean values of FreeMind, TuxGuitar, and jEdit equal to $0.79,0.42$, and 0.87 , respectively. These values are below 0.88 . We may explain this result by the fact that in [15], the DIT mean values are given per app, and in our case, per class. Although we note that according to [1], the DIT mean value of TuxGuitar equals to 1.46 and is in the range of acceptable values. Besides, in [15] the DIT mean values of native versions of FreeMind 0.8.1 and TuxGuitar 1.3.0 are 1.69 and 0.95, respectively. The 1.69 value is good and 0.95 value is acceptable. Only for the versions of jEdit 2.6final and 3.6final the DIT mean values are below 0.88 and equal to 0.63 and 0.42 , respectively.

The DIT mean values of native versions of FreeMind and TuxGuitar indicate the confidence to proposed bounds of the DIT mean intervals as the complexity indicators of OOD of open-source apps developed in Java.

\section{Discussion}

The paper is founded on the assumption that the DIT mean could be viewed as an app-level metric. This assumption is based on the papers of other authors, in particular [14], [15]. In [15] mean values of 16 software metrics (including the DIT mean) were considered to check their consistency across three apps and their versions. According to [14], the AID metric, that calculated as the sum of the depth of each class divided by the number of classes could be used to determine the complexity of a program as a whole. Also, like the AID metric at an app level, the DIT mean may be applied. However, the recommended values for the DIT mean of an app are not known. To find the recommended values for the 
DIT mean of an app we have proposed to use the confidence and prediction intervals. A DIT mean value of an app from the confidence interval is good since this interval indicates how reliable the estimate is for the DIT mean values of all apps used for estimating the interval with a certain significance level. We used 0.05 significance level as the appointed one usually although this value may be discussed. A DIT mean value between the lower bounds of the confidence and prediction intervals, or between the upper bounds of the confidence and prediction intervals is also acceptable.

We apply normalizing transformations to estimate the confidence and prediction intervals of the DIT mean by appropriate techniques [16] since the distribution of the DIT mean values is not Gaussian what the chi-squared test result and the values of estimators of skewness and kurtosis indicate. As stated earlier, estimators of skewness and kurtosis equal to 1.36 and 6.49 , respectively for the distribution of the DIT mean values. Moreover, the distributions of the DIT values for all from reviewed apps are not Gaussian what the chisquared test results and the values of estimators of skewness and kurtosis indicate too. For example, for 1390 DIT values of the jEdit app version 5.5.0, the chi-squared test statistic $\chi^{2}$ value equals to 972.92 is higher than the critical value of the chi-square, which equals to 12.59 for 6 degrees of freedom and 0.05 significance level, and estimators of skewness and kurtosis equal to 6.93 and 76.07 , respectively.

With respect to the proposed values of the bounds of the confidence and prediction intervals of the DIT mean as the complexity indicators of OOD of software apps from the point of view of the class hierarchy layout two limitations should be acknowledged and addressed concerning the data sample from 101 open-source apps developed in Java. The first limitation concerns the estimation of the data sample for open-source apps developed in Java only. The evaluation of other data samples, for example, for industrial apps in Java may affect the bounds of the confidence and prediction intervals of the DIT mean. In such cases, the proposed values of the bounds of the confidence and prediction intervals of the DIT mean remain to be confirmed or changed.

The second limitation concerns the sample size that equals 101 . This value can be unambiguously considered as the lower size limit of the large sample. Larger sample sizes may lead to a reduction of the widths of the confidence and prediction intervals.

It should also be noted that the complexity of OOD of software apps from the point of view of the class hierarchy layout may depend on other metrics, for example, the average number of children per class, the influence of which needs to be investigated.

\section{Conclusions}

We have proposed to apply the confidence and prediction intervals of the DIT mean as the complexity indicators of OOD of software apps from the point of view of the class hierarchy layout. For estimating the confidence and prediction intervals of the DIT mean is need to use normalizing transformations. In this case, we have used four transformations, such as the Box-Cox, Johnson for $S_{\mathrm{L}}, S_{\mathrm{B}}$, and $S_{\mathrm{U}}$ families. All these transformations have led to a similar normalization of the data sample in our case. The confidence and prediction intervals of the DIT mean using normalizing transformations for the data sample from 101 open-source apps developed in Java hosted on GitHub are estimated. The values of confidence and prediction interval bounds are similar for all four transformations. But only 
the Johnson transformation for $S_{\mathrm{U}}$ family is the bijective one. Therefore, we have selected the confidence and prediction intervals of the DIT mean for a significance level of 0.05 based on the Johnson transformation for $S_{U}$ family. In this case, a DIT mean value from 1.57 to 1.72 is good. A DIT mean value from 0.88 to 1.57 or from 1.72 to 4.47 is also acceptable. A DIT mean value below 0.88 may indicate poor exploitation of the advantages of OOD and inheritance. A DIT mean value higher than 4.47 may indicate that some classes have a large number of the inheritance levels from the object hierarchy top. Comparison with the DIT mean values of two popular open-source apps developed in Java illustrates the applicability of the proposed complexity indicators in the form of the confidence and prediction intervals of the DIT mean. In the future, to validate the complexity indicators of OOD of software apps from the point of view of the class hierarchy layout based on the confidence and prediction intervals of the DIT mean, further research needs to be carried out for other data sets, including apps defined type only, for example, frameworks. Also, the investigation of other metrics, for instance, the average number of children per class that affects the class hierarchy layout should be done.

\section{References}

[1] Barkmann, H., Lincke, R., Lowe, W., Quantitative evaluation of software quality metrics in open-source projects, in: Proceedings of the 2009 International Conference on Advanced Information Networking and Applications Workshops, Bradford, UK, 2009, 1067-1072. https://doi.org/10.1109/WAINA.2009.190

[2] Bouktif, S., Sahraoui, H., Ahmed, F., Predicting stability of open-source software systems using combination of Bayesian classifiers, ACM Transactions on Management Information Systems, 5, 1, Article 3, 2014, 1-26. https://doi.org/10.1145/2555596

[3] Bousquet, L.d., Shaheen, M.R., Relation between depth of inheritance tree and number of methods to test, in: Proceedings of the 1st International Conference on Software Testing, Verification, and Validation, Lillehammer, Norway, 2008, 161-170. https://doi.org/10.1109/ICST.2008.34

[4] Box, G.E.P., Cox, D.R., An analysis of transformations. Journal of the Royal Statistical Society. Series B (Methodological), 26, 2, 1964, 211-252.

[5] Chidamber, S.R., Kemerer, C.F., A metrics suite for object oriented design. IEEE Transactions on Software Engineering, 20, 6, 1994, 476-493. http://dx.doi.org/10.1109/32.295895

[6] Depth of Inheritance Tree, https://www.cachequality.com/docs/metrics/depthinheritance-tree, last accessed 2020/04/16.

[7] Elahi, E., Kanwal, S., Asif, A.N., A new ensemble approach for software fault prediction, in: Proceedings of the 17th International Bhurban Conference on Applied Sciences and Technology (IBCAST), Islamabad, Pakistan, 2020, 407-412. https://doi.org/10.1109/IBCAST47879.2020.9044596 
[8] Foucault, M., Teyton, C., Lo, D., Blanc, X., Falleri, J.R., On the usefulness of ownership metrics in open-source software projects, Information and Software Technology, 64, 2015, 102-112. https://doi.org/10.1016/j.infsof.2015.01.013

[9] Freedman, D., Pisani, R., Purves, R., Statistics. 4th edn. Wiley, 2007.

[10] Grubbs, F., Procedures for detecting outlying observations in samples, Technometrics, 11, 1, 1969, 1-21.

[11] Johnson, R.A., Wichern, D.W., Applied multivariate statistical analysis, Pearson Prentice Hall, 2007.

[12] Kendall, M.G., Stuart, A., The advanced theory of statistics. Vol. 1, Distribution Theory. 2nd edn., Charles Griffin \& Company Limited, London, 1963.

[13] Makkar, G., Chhabra, J.K., Challa, R.K., Object oriented inheritance metric-reusability perspective, in: Proceedings of the International Conference on Computing, Electronics and Electrical Technologies (ICCEET), Kumaracoil, India, 2012, 852-859. https://doi.org/10.1109/ICCEET.2012.6203815

[14] Mishra, D., New Inheritance Complexity Metrics for Object-Oriented Software Systems: An Evaluation with Weyuker's Properties, Computing and Informatics, 30, 2, 2011, 267-293.

[15] Molnar AJ., Neamţu A., Motogna S., Evaluation of software product quality metrics, in: Damiani E., Spanoudakis G., Maciaszek L. (eds.), Evaluation of Novel Approaches to Software Engineering. ENASE 2019. Communications in Computer and Information Science, vol. 1172, Springer, Cham, 2020, 163-187. https://doi.org/10.1007/978-3-03040223-5_8

[16] Prykhodko, S.B., Statistical anomaly detection techniques based on normalizing transformations for non-Gaussian data, in: Proceedings of the International Conference on Computational Intelligence (Results, Problems and Perspectives), Kyiv-Cherkasy, Ukraine, 2015, 286-287.

[17] Prykhodko, S., Prykhodko, N., Makarova, L., Pugachenko, K., Detecting outliers in multivariate non-Gaussian data on the basis of normalizing transformations, in: Proceedings of the 2017 IEEE First Ukraine Conference on Electrical and Computer Engineering (UKRCON), Kyiv, Ukraine, 2017, 846-849. https://doi.org/10.1109/UKRCON.2017.8100366

[18] Prykhodko, N., Prykhodko, S., Vorona, M., The non-linear regression model to estimate the part of NPLS in the whole loan portfolio of Ukrainian banks, in: Proceedings of the 2018 IEEE First International Conference on System Analysis \& Intelligent Computing (SAIC), Kyiv, Ukraine, 2018, 261-265. https://doi.org/10.1109/SAIC.2018.8516899

[19] Rathore, S.S., Kumar, S., A study on software fault prediction techniques, Artificial Intelligence Review, 51, 2, 2019, 255-327. https://doi.org/10.1007/s10462-017-9563-5 
[20] Shaheen, M.R., Bousquet, L.d., Is depth of inheritance tree a good cost prediction for branch coverage testing? in: Proceedings of the First International Conference on Advances in System Testing and Validation Lifecycle, Porto, Portugal, 2009, $42-47$. https://doi.org/10.1109/VALID.2009.11

[21] Shatnawi, R., Empirical study of fault prediction for open-source systems using the Chidamber and Kemerer metrics, IET Software, 8, 3, 2014, 113-119. http://dx.doi.org/10.1049/iet-sen.2013.0008

Received 14.07.2020, Accepted 10.01.2021 